Article

\title{
Adaptive Control for Pure-Feedback Nonlinear Systems Preceded by Asymmetric Hysteresis
}

\author{
Xiaohuan Lai, Haipeng Pan * and Xinlong Zhao \\ Faculty of Mechanical Engineering and Automation, Zhejiang Sci-Tech University, Hangzhou 310018, China; \\ xiaohuanlai@163.com (X.L.); zhaoxinlong@zstu.edu.cn (X.Z.) \\ * Correspondence: pan@zstu.edu.cn
}

Received: 8 November 2019; Accepted: 3 December 2019; Published: 9 December 2019

\begin{abstract}
An adaptive control scheme is proposed for a class of uncertain pure-feedback nonlinear systems preceded by asymmetric hysteresis nonlinearity. The asymmetric property is described by the modified Bouc-Wen model based on the proposed asymmetric factor. State variables in the controller design are directly replaced with nonaffine functions to address the control problem caused by nonaffine appearance. Moreover, the control method can handle systems with external disturbances and guarantee the global stability of all the signals in the closed-loop system. The feasibility of the control scheme is verified by a simulation example and experimental results.
\end{abstract}

Keywords: adaptive control; pure-feedback system; asymmetric hysteresis; nonaffine functions

\section{Introduction}

Piezoelectric actuators have the advantages of high position resolution (nanometers or below), maximum blocking force (around hundreds of $N$ ), high stiffness, wide bandwidth, and very short response time and play a key role in precision manufacturing and measurement [1]. However, the major disadvantage of piezoelectric materials involves their inherent hysteresis nonlinearities, which could dramatically degrade system performances and may even cause instability [2]. The hysteresis behavior of piezoelectric actuators tends to be asymmetric in practical applications [3,4]. Various hysteresis models have been proposed to describe the characteristics of hysteresis, including the Preisach model, Krasnosel'skii-Pokrovkii model, Prandtl-Ishlinskii model, the Duhem model, Backlash-like model, Bouc-Wen model [5]. In these models, Preisach model, KP model and Duhem model are generally unable to describe asymmetric hysteresis. In order to describe the asymmetric property, the modified Backlash-like model is proposed [6]. However, the Prandt-Ishlinkii model [7-10] and the Bouc-Wen model [11-13] are the most popular modified models used to address asymmetric hysteresis nonlinearity. The asymmetric Prandt-Ishlinkii model is generally realized through the modification of the generalized play operator with asymmetry [9]. However, the disadvantage of this method is that it generally increases the complexity of the mathematical form of the operator and the difficulty of parameter identification and controller design. The asymmetric Bouc-Wen model is generally obtained through the transformation of an intermediate variable with an asymmetric property $[11,12]$. However, this approach comes, at the expense of identification complexity as the number of parameters increases. In fact, the above information shows that asymmetric hysteresis is far more complex than general symmetric hysteresis.

Given that hysteresis is inevitable in actual systems, the control of nonlinear systems with hysteresis has received considerable attention and numerous achievements have been attained in recent years [14-23]. Hysteresis inverse methods have been proposed to mitigate the effect of hysteresis in nonlinear systems $[17,19,24-26]$. Robust adaptive control and neural control have been directly applied to compensate for hysteresis without the construction of an inverse model [27-30]. In these 
methods, the neural network is used to approximate the unknown nonlinear function. Thus, the neural network is incorporated with controller for hysteresis compensation. However, most of these control methods are valid only for strict-feedback systems[20,27,28,30-33]. These methods cannot be applied directly to pure-feedback systems.

The cascade and nonaffine properties of pure-feedback systems complicate the determination of explicit virtual controls and the actual control to stabilize these systems. The mean value theorem [34-37] and Taylor series expansion [38,39] have been proposed to convert the nonaffine systems into their equivalent affine form to address the controller design in pure-feedback systems. Adaptive neural network control schemes and fuzzy control based on the mean-value theorem have been proposed for a class of uncertain pure-feedback nonlinear systems [40-43]. However, the mean value theorem method increases the complexity of the system and may cause the circular design problem [35-37]. Fuzzy control has been developed for nonaffine systems through the use of the Taylor series expansion method [44]. Nevertheless, this method may result in additional uncertainties in the system. The foregoing design of pure-feedback systems without consideration for hysteresis nonlinearity as a control input is extremely difficult. The control design will become highly challenging when pure-feedback systems are preceded by asymmetric hysteresis. Thus, this research topic involves theoretical difficulties and has practical importance.

In past decades, adaptive control has been applied in various complex nonlinear systems due to its excellent performance $[45,46]$. Motivated by the above conditions, this work adopts a novel backstepping adaptive control scheme for a class of pure-feedback nonlinear systems with asymmetric hysteresis nonlinearity. The advantages of the proposed approach are presented below.

1. The proposed model is simple and may present asymmetry through the embedding of an asymmetric factor in the classic Bouc-Wen model. Furthermore, the component of the hysteresis output in the proposed asymmetric Bouc-Wen model has been proven to be bounded. This characteristic is essential for the controller design.

2. The novel adaptive control scheme for pure-feedback systems is implemented through the use of nonaffine functions to replace state variables in the backstepping design. This method overcomes control problems caused by nonaffine appearance. Furthermore, it simplifies the control scheme and ensures the global stability of all closed-loop signals. The feasibility and effectiveness of the adopted control design are demonstrated through simulation and experimental works in dSPACE.

The rest of the paper is organized as follows. The problem formulation and preliminaries for the uncertain pure-feedback system are presented in Section 2. We propose the adaptive backstepping design procedure of the pure-feedback system in the presence of disturbances and provide the stability analysis for the closed-loop system in Section 3. Section 4 demonstrates the performance of the proposed control scheme through a simulation example and dSPACE experimental results. Finally, conclusions are drawn in Section 5. The detailed proof of the fact that $h$ is bounded is given in detail in Appendix A.

\section{Problem Formulation and Preliminaries}

Consider a class of uncertain pure-feedback nonlinear systems with asymmetric hysteresis characteristics described by the $n$th order differential equations of the form:

$$
\begin{aligned}
& \dot{x}_{i}=\theta_{i} f_{i}\left(\bar{x}_{i}\right)+g_{i}\left(\bar{x}_{i}, x_{i+1}\right)+d_{i}, i=1,2, \cdots, n-1 \\
& \dot{x}_{n}=\theta_{n} f_{n}\left(\bar{x}_{i}\right)+b u(v)+d_{n}, n \geq 2 \\
& y=x_{1}
\end{aligned}
$$

where $\bar{x}_{i}=\left[x_{1}, x_{2}, \cdots, x_{i}\right]^{T} \in R^{i}, i=1,2, \cdots, n-1$ is the vector of states of the $i$ th equations, $\bar{x}_{n}=\left[x_{1}, x_{2}, \cdots, x_{n}\right]^{T} \in R^{n} ; y=x_{1} \in R$ is the output, $\theta_{i}, i=1,2, \cdots, n$ and $b \neq 0$ are unknown constant parameters, and $d_{i}, i=1,2, \cdots, n$ are unknown bounded disturbance functions. $f_{i}\left(\bar{x}_{i}\right), f_{n}\left(\bar{x}_{n}\right)$ 
and $g_{i}\left(\bar{x}_{i}, x_{i+1}\right)$ are known smooth functions, designated as $f_{i}, f_{n}$ and $g_{i}$ respectively in the following passage for simplicity.

Given the difficulties to control nonaffine systems, the systems considered in this paper are affine in control $u$ but nonaffine in state $x_{i}$ in the first $n-1$ steps represented by the nonaffine functions $g_{i}$. $u(v)$ donotes asymmetric hysteresis nonlinearity, which can be described in the form of the proposed asymmetric Bouc-Wen model as follows:

$$
\begin{gathered}
u=d_{p} v+h \\
\dot{h}=\dot{v}\left(A_{b w}-|h|^{m}(\beta \operatorname{sgn}(\dot{v} h)+\gamma)\right)+\delta \dot{v} \operatorname{sgn}(v)
\end{gathered}
$$

where, $v$ and $u$ are the input and output of hysteresis behavior, respectively, $h$ is the component of the hysteresis output, $A_{b w}>0$ and $m \geq 1$ are the parameters that control the scale and sharpness of the hysteresis loop, and $\beta$ and $\gamma$ are other shape features of the hysteresis loop of $\dot{v}$ and $h$ and satisfy $\beta+\gamma>0, \beta-\gamma \geq 0 . \dot{v} \operatorname{sgn}(v)$ is introduced to present the asymmetric property, and $\delta<0$ is the asymmetric factor and satisfy $|\delta|<\left|A_{b w}\right|$. Furthermore, $h$ has been proven to be bounded in appendix, which is essential for the controller design with guaranteed stability. And parameters $d_{p}, A_{b w}, \beta, \gamma, m$ can be obtained by particle swarm optimization and hybrid evolutionary algorithm $[47,48]$.

Equation (3) can be used to model aymmetric hysteresis, as shown in Figure 1, with $d_{p}=1.5$, $A_{b w}=1, \beta=2, \gamma=1.8, m=1, \delta=-0.3$ in our design, and the input signal is $v(t)=\sin (t)$.

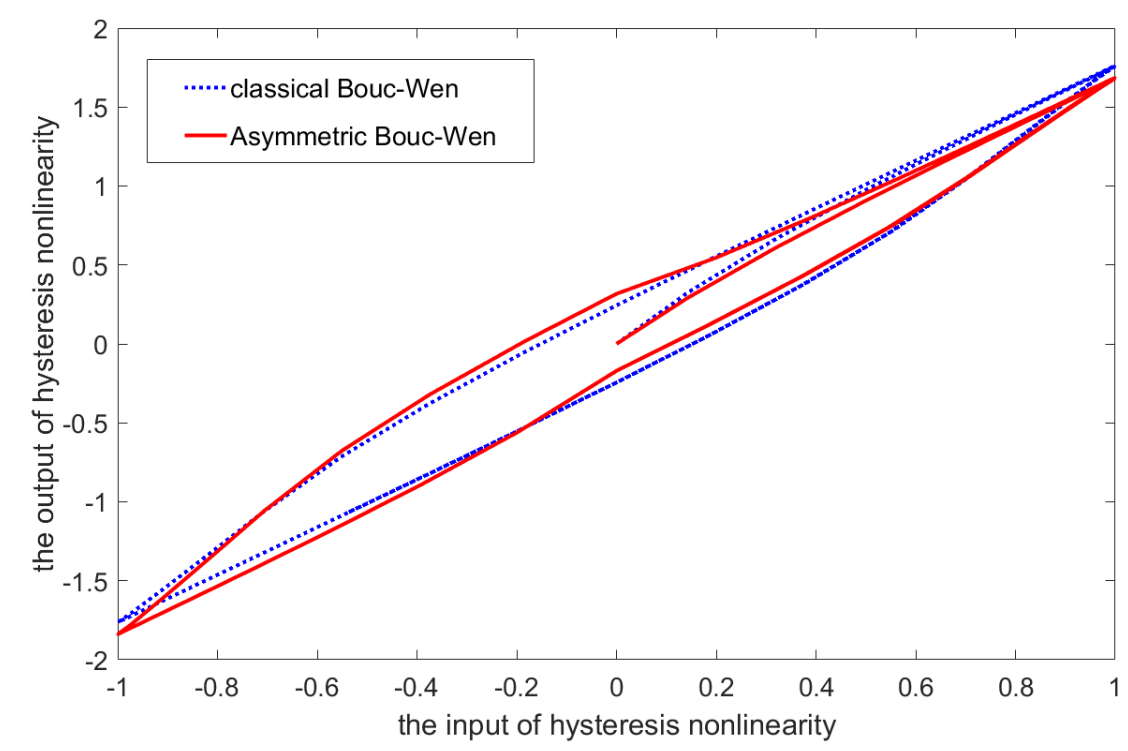

Figure 1. Curve of classical Bouc-Wen with $\delta=0$ and asymmetric Bouc-Wen with $\delta=-0.3$.

Remark 1. Figure 1 shows that large absolute value of $\delta$ may correspond to the pronounced asymmetry of the hysteresis.

Through the application of (2), the original system can be rewritten as

$$
\begin{aligned}
& \dot{x}_{i}=\theta_{i} f_{i}\left(\bar{x}_{i}\right)+g_{i}\left(\bar{x}_{i}, x_{i+1}\right)+d_{i}, i=1,2, \cdots, n-1 \\
& \dot{x}_{n}=\theta_{n} f_{n}\left(\bar{x}_{i}\right)+p v+\bar{d}_{n}, n \geq 2 \\
& y=x_{1}
\end{aligned}
$$

where $p=b d_{p}$ and $\bar{d}_{n}=b h+d_{n}$, and the hysteresis input $v$ can be viewed as the new control input.

The control object is to design the adaptive controller $v$ to ensure that all closed loop signals are bounded, and the output $y$ tracks a given reference signal $y_{d}$ under the following assumptions. 
Assumption 1: A positive constant $\varepsilon$ exists such that $\left\|\frac{\partial g_{i}\left(\bar{x}_{i}, x_{i+1}\right)}{\partial x_{i+1}}\right\| \geq \varepsilon>0, i=1,2, \cdots, n-1$.

Assumption 2: The unknown parameters $b$ and $d_{p}$ satisfy $p=b d_{p}>0$.

Assumption 3: The desired trajectory $y_{d}$ and its $n$th order derivatives are known and bounded.

Assumption 4: All disturbances own their boundaries, that is, $\left\|d_{i}\right\| \leq \chi_{i} \leq \omega_{i}^{*} \rho_{i},\left\|\bar{d}_{n}\right\| \leq \chi_{n} \leq \omega_{n}^{*} \rho_{n}$. Parameter values $\omega_{i}^{*} \geq 0, \omega_{n}^{*}>0$ and known smooth functions, $\rho_{i}$ and $\rho_{n}$ exist.

\section{The Controller Design and Stability Analysis}

The standard backstepping method selects state variables as the virtual control variable of each subsystem, which can solve the control design of the strict-feedback systems effectively. However, the main feature of pure-feedback nonlinear systems namely the system states or control inputs always appear implicitly in the nonaffine functions, which often makes the explicit virtual and actual control laws difficult to obtain. As a result, the standard backstepping is inapplicable in pure-feedback systems. State variables are directly replaced with nonaffine functions at each step to deal with pure-feedback systems as in (1). The above replacement is the major difference compared with standard backstepping method. Thus, the transformation from pure-feedback systems to strict-feedback systems could be avoided. Transformation methods, such as the mean value theorem and Taylor series expansion, are not required. Therefore, the proposed method may avoid additional uncertainty and the circular design problem.

The novel backstepping-based control procedure contains $n$ recursive steps and involves the following change of coordinates:

$$
\begin{aligned}
& z_{1}=y-y_{d} \\
& z_{i}=g_{i-1}-\alpha_{i-1}, i=2,3, \cdots, n
\end{aligned}
$$

where $\alpha_{i-1}$ is the virtual control law at the $i$ th step.

An outline of the novel backstepping design procedure for system (1) is given below.

Step 1:

Given that $z_{1}=x_{1}-y_{d}$ and $z_{2}=g_{1}-\alpha_{1}$, the derivative of $z_{1}$ is

$$
\dot{z}_{1}=\theta_{1} f_{1}+z_{2}+\alpha_{1}+d_{1}-\dot{y}_{d}
$$

The virtual control law $\alpha_{1}$ and the adaptive law for adjusting $\omega_{1}$ are designed as

$$
\begin{aligned}
\alpha_{1} & =\tilde{\alpha}_{1}-\varphi_{1} \\
\tilde{\alpha}_{1} & =-k_{1} z_{1}-\hat{\theta}_{1} f_{1}+\dot{y}_{d} \\
\varphi_{1} & =\omega_{1} \rho_{1} \tanh \left(\frac{z_{1} \rho_{1}}{\mathcal{K}_{1}}\right) \\
\dot{\omega}_{1} & =\varsigma_{1}\left[z_{1} \rho_{1} \tanh \left(\frac{z_{1} \rho_{1}}{\mathcal{K}_{1}}\right)-\sigma_{1}\left(\omega_{1}-\omega_{1}^{0}\right)\right]
\end{aligned}
$$

In $\tilde{\alpha}_{1}, k_{1}$ is a positive constant, and $\hat{\theta}_{1}$ is the estimate of $\theta_{1}$. In $\varphi_{1}, \omega_{1}$ and $\rho_{1}$ satisfy Assumption 4 , and $\dot{\omega}_{1}$ is the adjusting law of $\omega_{1}$.

Remark 2. Consider the existence of disturbances and asymmetric hysteresis behavior, and the design of the virtual control law $\alpha_{i}$ and the adaptive control law $\bar{v}$ changes to $\alpha_{i}=\tilde{\alpha}_{i}-\varphi_{i}$ and $\bar{v}=\tilde{v}-\varphi_{n}$, respectively. $\tilde{\alpha}_{i}$ and $\tilde{v}$ are designed in accordance with the backstepping procedure, and the influence of uncertain disturbance items $d_{i}$ and $\bar{d}_{n}$ is eliminated through the use of $\varphi_{i}$ and $\varphi_{n}$.

Define the Lyapunov function $V_{1}$ as

$$
V_{1}=\frac{1}{2} z_{1}^{2}+\frac{1}{2} \tilde{\theta}_{1} \Lambda^{-1} \tilde{\theta}_{1}+\frac{1}{2 \varsigma_{1}} \chi_{1}^{2}
$$


where $\tilde{\theta}_{1}=\theta_{1}-\hat{\theta}_{1}, \Lambda$ is a symmetric positive definite matrix, and $\chi_{1}=\omega_{1}-\omega_{1}^{M}$ and $\omega_{1}^{M}=$ $\max \left(\omega_{1}^{*}, \omega_{1}^{0}\right)$.

Thus, the derivative of $V_{1}$ can be obtained as follows

$$
\begin{aligned}
\dot{V}_{1}= & z_{1} \dot{z}_{1}+\tilde{\theta}_{1} \Lambda^{-1} \dot{\theta}_{1}+\frac{1}{\zeta_{1}} \chi_{1} \dot{\chi}_{1} \\
= & z_{1}\left(\theta_{1} f_{1}+z_{2}+\alpha_{1}+d_{1}-\dot{y}_{d}\right)-\tilde{\theta}_{1} \Lambda^{-1} \dot{\hat{\theta}}_{1}+\frac{1}{\varsigma_{1}} \chi_{1}\left(\varsigma_{1}\left[z_{1} \rho_{1} \tanh \left(\frac{z_{1} \rho_{1}}{\mathcal{K}_{1}}\right)-\sigma_{1}\left(\omega_{1}-\omega_{1}^{0}\right)\right]\right) \\
= & z_{1}\left(\theta_{1} f_{1}+z_{2}+\tilde{\alpha}_{1}-\varphi_{1}+d_{1}-\dot{y}_{d}\right)-\tilde{\theta}_{1} \Lambda^{-1} \hat{\theta}_{1}+\chi_{1}\left[z_{1} \rho_{1} \tanh \left(\frac{z_{1} \rho_{1}}{\mathcal{K}_{1}}\right)-\sigma_{1}\left(\omega_{1}-\omega_{1}^{0}\right)\right] \\
= & z_{1}\left(\theta_{1} f_{1}+z_{2}-k_{1} z_{1}-\hat{\theta}_{1} f_{1}-\varphi_{1}+d_{1}\right)-\tilde{\theta}_{1} \Lambda^{-1} \dot{\hat{\theta}}_{1} \\
& +\left(\omega_{1}-\omega_{1}^{M}\right)\left[z_{1} \rho_{1} \tanh \left(\frac{z_{1} \rho_{1}}{\mathcal{K}_{1}}\right)-\sigma_{1}\left(\omega_{1}-\omega_{1}^{0}\right)\right] \\
= & z_{1}\left(\tilde{\theta}_{1} f_{1}+z_{2}-k_{1} z_{1}-\omega_{1} \rho_{1} \tanh \left(\frac{z_{1} \rho_{1}}{\mathcal{K}_{1}}\right)+d_{1}\right)-\tilde{\theta}_{1} \Lambda^{-1} \dot{\hat{\theta}}_{1} \\
& +\left(\omega_{1}-\omega_{1}^{M}\right)\left[z_{1} \rho_{1} \tanh \left(\frac{z_{1} \rho_{1}}{\mathcal{K}_{1}}\right)\right]-\chi_{1} \sigma_{1}\left(\omega_{1}-\omega_{1}^{0}\right) \\
= & -k_{1} z_{1}^{2}+z_{1} z_{2}+\tilde{\theta}_{1} \Lambda^{-1}\left(\tau_{1,1}-\dot{\theta}_{1}\right)+z_{1} d_{1}-z_{1} \omega_{1}^{M} \rho_{1} \tanh \left(\frac{z_{1} \rho_{1}}{\mathcal{K}_{1}}\right)-\chi_{1} \sigma_{1}\left(\omega_{1}-\omega_{1}^{0}\right)
\end{aligned}
$$

where $\tau_{1,1}=\Lambda f_{1} z_{1}, \dot{\hat{\theta}}_{1}=\tau_{1,1}-\Lambda \sigma_{\theta}\left(\hat{\theta}_{1}-\theta_{1}^{0}\right)$ and $\sigma_{\theta}>0$ is a positive constant, and $\theta_{1}^{0}$ is a design constant.

The subsequent recursive backstepping steps will be omitted, similar to that in Step 1 . The final controller design is summarized in Table 1.

Theorem 1. Consider the uncertain pure-feedback nonlinear systems (1) that satisfy Assumptions 1-4. All the signals in the closed-loop system are globally bounded with the application of the controller (T-3) and the adaptive laws (T-12)-(T-15).

Proof: We define the Lyapunov function $V_{n}$ as

$$
V_{n}=V_{n-1}+\frac{1}{2} \tilde{\theta}_{n} \Lambda^{-1} \tilde{\theta}_{n}+\frac{1}{2} z_{n}^{2}+\frac{p}{2 \eta} \tilde{\phi}^{2}+\frac{1}{2 \varsigma_{n}} \chi_{n}^{2}
$$

Note that $0 \leq|\mu|-\mu \tanh \left(\frac{\mu}{\epsilon}\right) \leq \xi \mathcal{E}$ holds for any $\varepsilon>0$, and any $\mu \in \mathbb{R}$ and $\xi$ satisfy $\xi=e^{-(\xi+1)}$, that is, $\xi=0.2785[49]$.

From the (T-3)-(T-11) controllers and the (T-12)-(T-15) parameter estimates, the derivative of the Lyapunov function satisfies

$$
\begin{aligned}
\dot{V}_{n} \leq & -\sum_{j=1}^{n} k_{j} z_{j}^{2}+\frac{\sigma_{\theta}}{2} \sum_{j=1}^{n} \tilde{\theta}_{j}^{2}+\sum_{j=1}^{n} \frac{\sigma_{j}}{2} \chi_{j}^{2}+\frac{1}{2 \sigma_{\theta}} \sum_{j=1}^{n}\left(\hat{\theta}_{j}-\theta_{j}^{0}\right)^{2}+\sum_{j=1}^{n} \frac{1}{2 \sigma_{j}}\left(\omega_{j}^{0}-\omega_{j}\right)^{2} \\
& +\sum_{j=1}^{n} \frac{1}{2} \omega_{j}^{M} \mathcal{K}_{j}+\sum_{j=2}^{n} \frac{1}{2} \frac{\omega_{j}^{M} \rho_{j}}{\omega_{n}^{M} \rho_{n}} \mathcal{K}_{n} \\
& \leq-c_{n} V_{n}+\lambda_{n}
\end{aligned}
$$

where

$$
\begin{aligned}
& c_{n}=\min \left\{2 k_{1}, \cdots, 2 k_{n}, \frac{\sigma_{n}}{\lambda_{\min }\left(\Lambda^{-1}\right)}, \varsigma_{1} \sigma_{1}, \cdots, \varsigma_{n} \sigma_{n}\right\}>0 \\
& \lambda_{n}=\frac{1}{2 \sigma_{\theta}} \sum_{j=1}^{n}\left(\hat{\theta}_{j}-\theta_{j}^{0}\right)^{2}+\sum_{j=1}^{n} \frac{1}{2} \omega_{j}^{M} \mathcal{K}_{j}+\sum_{j=1}^{n} \frac{1}{2 \sigma_{j}}\left(\omega_{j}^{0}-\omega_{j}\right)^{2}+\sum_{j=2}^{n} \frac{1}{2} \frac{\omega_{j}^{M} \rho_{j}}{\omega_{n}^{M} \rho_{n}} \mathcal{K}_{n}
\end{aligned}
$$




$$
V_{n} \leq V_{n}(0) e^{-c_{n} t}+\frac{\lambda_{n}}{c_{n}}\left(1-e^{-c_{n} t}\right)
$$

is obtained in accordance with the exponential stability theorem [50] and direct integrations of (9).

Therefore, $V_{n}$ is bounded, which implies that $z_{1}, z_{2}, \cdots, z_{n}, \hat{\theta}_{1}, \hat{\theta}_{2}, \cdots, \hat{\theta}_{n}, \hat{\phi}$ and $\hat{\chi}_{n}$ are globally bounded. $z_{1}$ is bounded, hence $x_{1}, \alpha_{1}, g_{1}$ are bounded. Assumption 1 and a previous work [51], state that $x_{2}$ is likewise bounded. Furthermore, $z_{2}$ is bounded, thereby implying that $\alpha_{2}$ is bounded. Therefore $g_{2}$ is bounded, which leads to $x_{3}$ being bounded. In summary, we can conclude that $x_{1}, x_{2}$, $\cdots, x_{n}, \alpha_{1}, \alpha_{2}, \cdots, \alpha_{n-1}$, and $\bar{v}, v$ are bounded.

Table 1. Adaptive backstepping control.

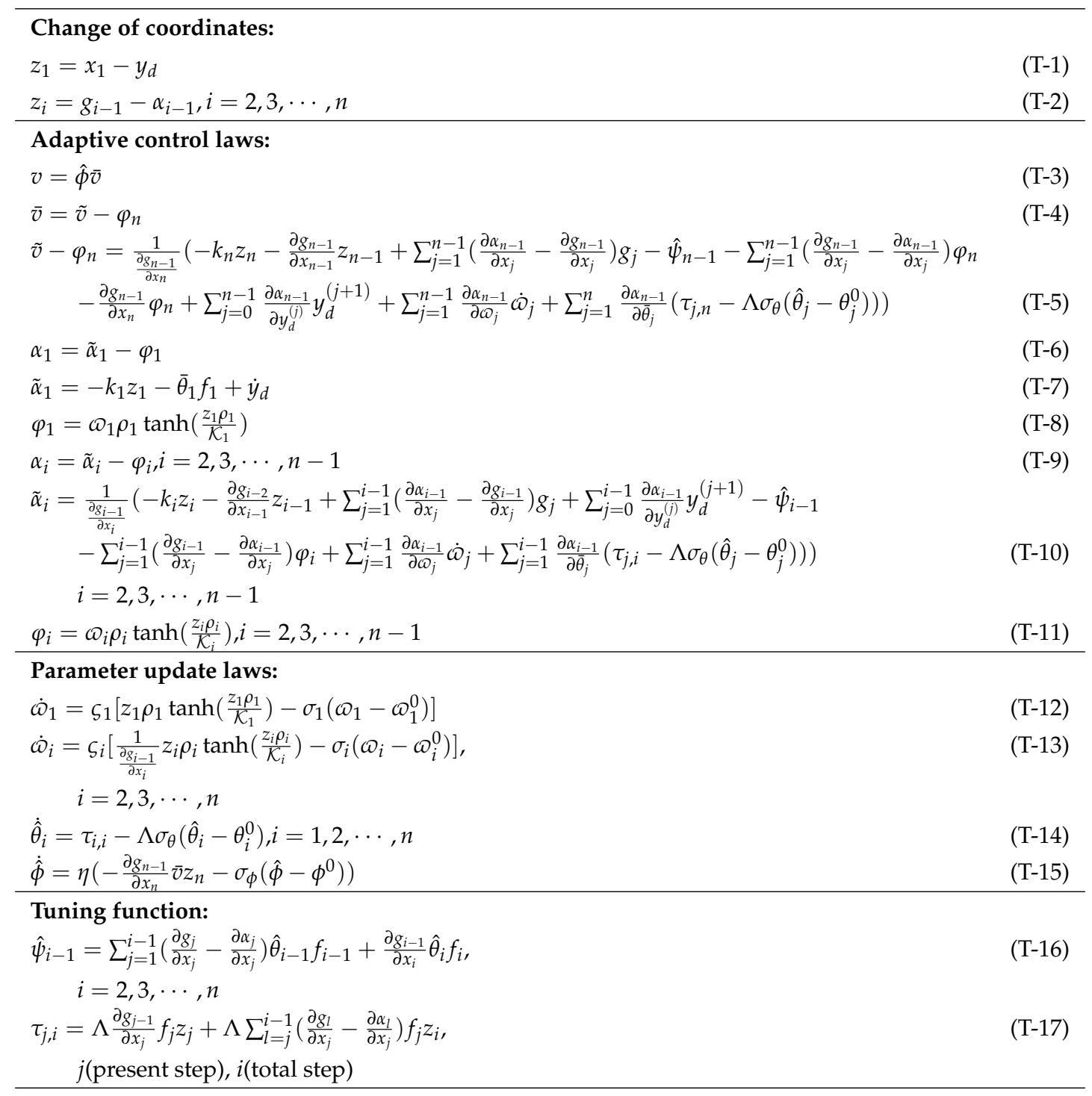

Remark 3. The main contributions in this paper are summarized as follows: (i) it is shown that the possibility for the proposed Bouc-Wen model as an illustration to hand asymmetric hysteresis nonlinearity; (ii) the difficulties from the nonaffine structures in pure-feedback systems are solved by a novel and simpler adaptive scheme implemented through the use of nonaffine functions to replace state variables in the backstepping design. 


\section{Simulation Example and Experimental Result}

A numerical simulation example is shown in this section to prove the feasibility of the control scheme. Furthermore, we conduct an experimental verification on the dSPACE platform to improve the reliability of the results for practical application.

\subsection{Simulation Example}

Consider pure-feedback nonlinear systems with external disturbances as follows:

$$
\begin{aligned}
& \dot{x}_{1}=\theta_{1} \frac{1-e^{-0.01 x_{1}}}{1+e^{-0.01 x_{1}}}+q\left(x_{2}^{3}+x_{2}\right)+d_{1} \\
& \dot{x}_{2}=\theta_{2} x_{1}^{2} x_{2}+b u(v)+d_{2} \\
& y=x_{1} \\
& u=d_{p} v+h \\
& \dot{h}=\dot{v}\left(A_{b w}-|h|^{m}(\beta \operatorname{sgn}(\dot{v} h)+\gamma)\right)+\delta \dot{v} \operatorname{sgn}(v)
\end{aligned}
$$

In this simulation, $\theta_{1}=15, q=8, \theta_{2}=0.5$, and $b=2$, and the disturbances are $d_{1}=0.1 \sin (t)$, and $d_{2}=0.25 \cos (t)$. Thus, $\omega_{1}^{*}=0.1, \rho_{1}=1, \omega_{2}^{*}=1.42$ and $\rho_{2}=1$. The initial values of the state are given as $x_{1}(0)=-1.2$ and $x_{2}(0)=0$. The desired tracking signal is $y_{d}=\sin (t)-2 \cos (0.5 t)$. The parameters of the modified Bouc-Wen model are chosen as $d_{p}=1.5, A_{b w}=1, \beta=2, \gamma=1.8$, $m=1$, and $\delta=-0.3$. The design parameters are selected as $k_{1}=k_{2}=1, \eta=1, \zeta_{1}=0.01$, $\phi^{0}=\omega_{1}^{0}=\omega_{2}^{0}=0, k_{1}=100$, and $k_{2}=100 . \Lambda$ is first order unit matrix, namely, $\Lambda=1$.

The adopted controller is compared with the control design in a previous work [19]. The obtained simulation results, depicted in Figures 2 and 3, show the effectiveness and advantage of the proposed controller over the reference controller [19]. The desired trajectory, the output of the proposed scheme and the output signal of the reference scheme [19] are shown in Figure 2. The error between the proposed scheme and the desired trajectory and the error between the reference scheme [19] and the desired trajectory are shown in Figure 3. The $E_{M A X}$ and $E_{R M S}$ of the proposed scheme and scheme in [19] are shown in Table 2. Compared with the reference method [19], the proposed method can accurately track the reference signal of a class of pure-feedback nonlinear in the presence of system uncertainty and external disturbances, thereby exhibiting stronger robustness. The proposed control law $v$ is illustrated in Figure 4.

In conclusion, an adaptive controller can modify its achieves in response to the dynamics in the process of systems and the characteristic of disturbances. And therefore, as the simulation time increases, its control performance will get better, which can be demonstrated from the above simulation results.

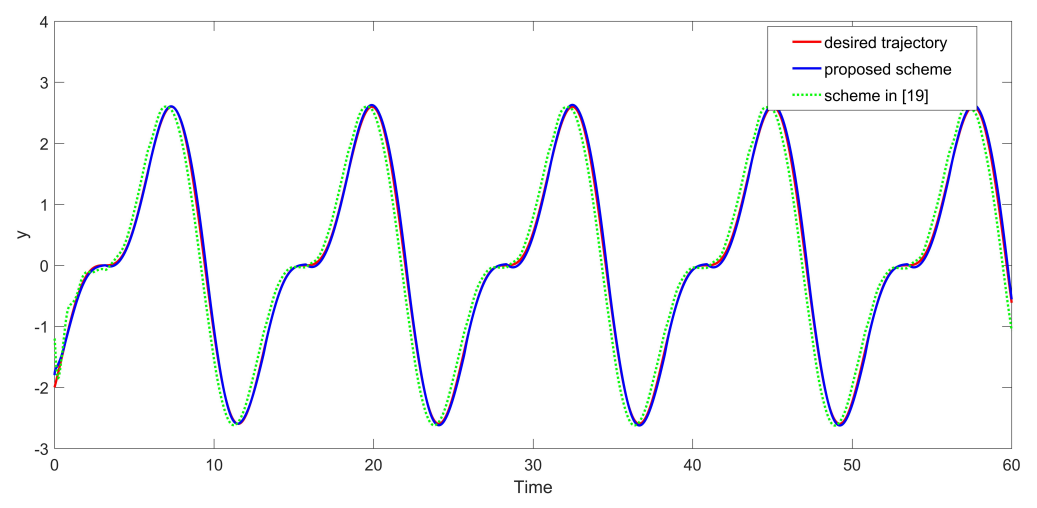

Figure 2. Simulation output-tracking performance. 


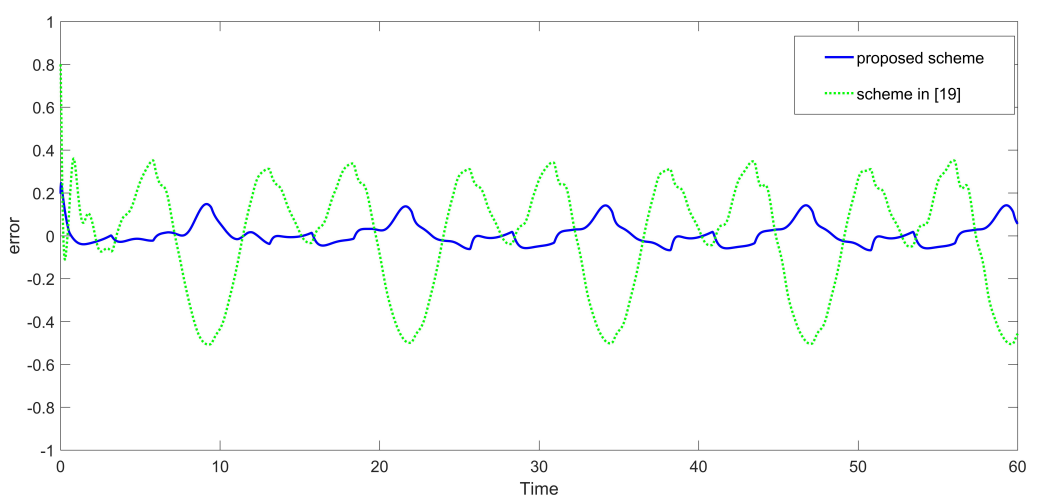

Figure 3. Error between the system and the desired trajectory.

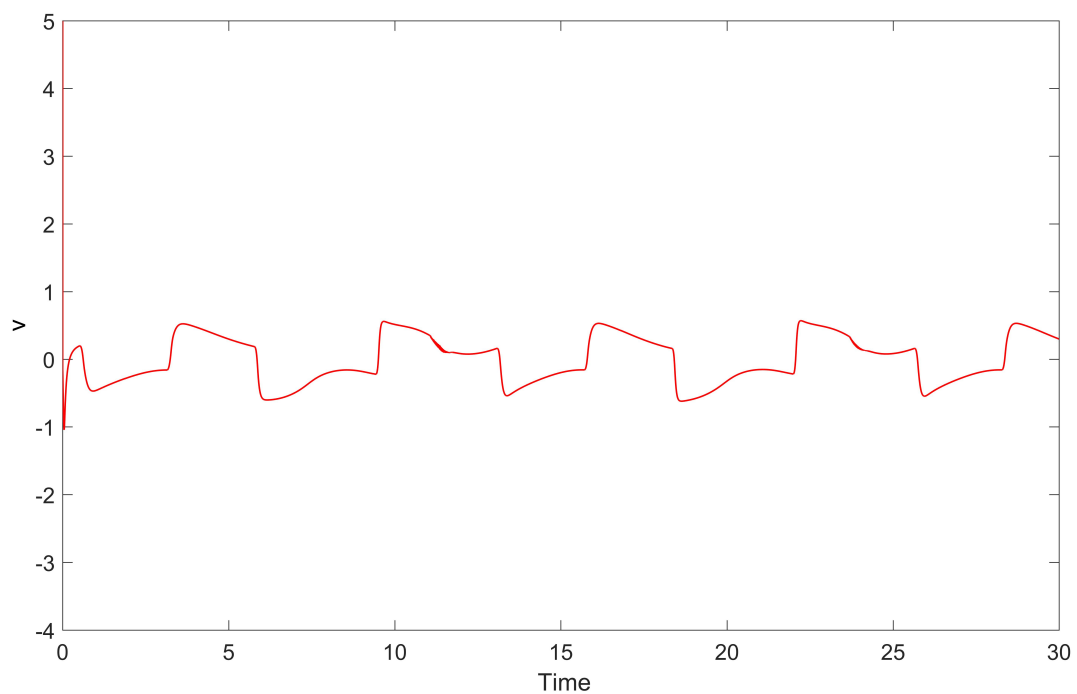

Figure 4. Control law $v$.

In order to compare an influence of the constant parameters in controller on the system output, the simulation results are carried out for different $k_{1}$ and $k_{2}$ as shown in Figures 5 and 6.

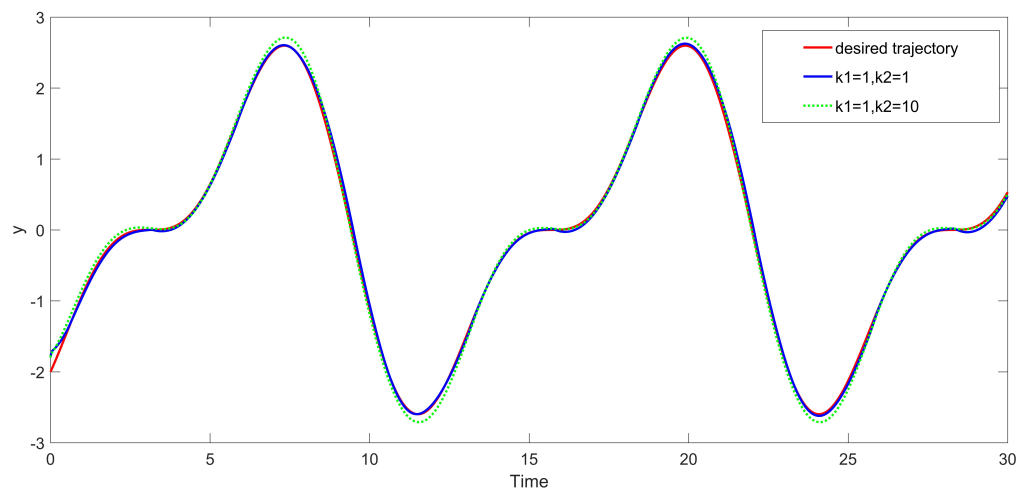

Figure 5. the influence of change of constant parameter $k_{2}$ impact on system output. 
Table 2. $E_{M A X}$ and $E_{R M S}$ of the proposed scheme and scheme in [19].

\begin{tabular}{ccc}
\hline Error & The Proposed Scheme & Scheme in [19] \\
\hline$E_{M A X}$ & 0.2456 & 0.8 \\
$E_{R M S}$ & 0.0524 & 0.5137 \\
\hline
\end{tabular}

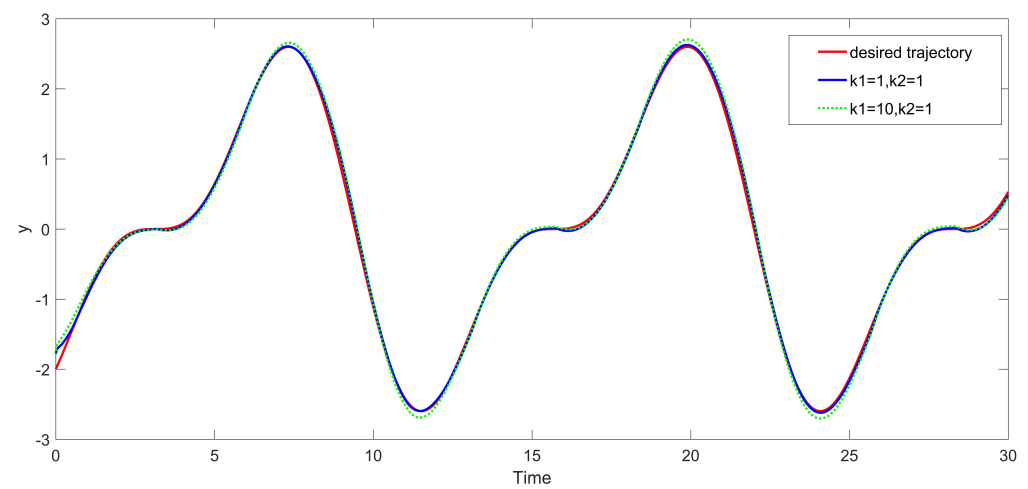

Figure 6. the influence of change of constant parameter $k_{1}$ impact on system output.

\subsection{Experimental Results}

Experiments are conducted on the piezoelectric actuated platform to verify the effectiveness of the proposed control scheme as shown in Figure 7. The experimental platform consists of the following elements.

1. Piezoelectric actuator: A PZT-752.21C piezoelectric actuator manufactured by Physik Instrument Company is utilized in this experiment. The actuator has a normal expansion of 0-30 $\mu \mathrm{m}$ under the input voltage $0-100 \mathrm{~V}$.

2. Voltage amplifier: A voltage amplifier (LVPZT, E-509) with a fixed gain of 10 is used as the excitation voltage of the regulation actuator.

3. Capacitive sensor: An integrated capacitive sensor is used to measure the displacement response of the actuator.

4. Data acquisition system: The dSPACE DS1103 control board is used to obtain the displacement of the piezoelectric actuator. The displacement is measured by a capacitive sensor. The dSPACE is equipped with a 16-bit analog-to-digital converter (ADC) and a 16-bit digital-to-analog converter (DAC).

In this experiment, pure-feedback nonlinear systems are same as the ones described in Section 4.1 and the desired tracking signal is $y_{d}=\sin (t)-2 \cos (0.5 t)$. The design parameters are selected as $\omega_{1}^{*}=0.1, \rho_{1}=1, \omega_{2}^{*}=1.42$ and $\rho_{2}=1, k_{1}=k_{2}=1, \eta=1, \zeta_{1}=0.01, \phi^{0}=\omega_{1}^{0}=\omega_{2}^{0}=0, k_{1}=100$, and $k_{2}=100 . \Lambda$ is first order unit matrix, namely, $\Lambda=1$. The initial values of the state are given as $x_{1}(0)=-1.2$ and $x_{2}(0)=0$.

The tracking of the experimental output and the desired trajectory are shown in Figure 8. The experimental data show satisfactory tracking performance, thereby demonstrating the effectiveness of the controller. Figure 9 shows that a minor difference exists between the error of the two systems, and the system has satisfactory stability.

A satisfactory agreement between the simulation and the experimental results is demonstrated. 


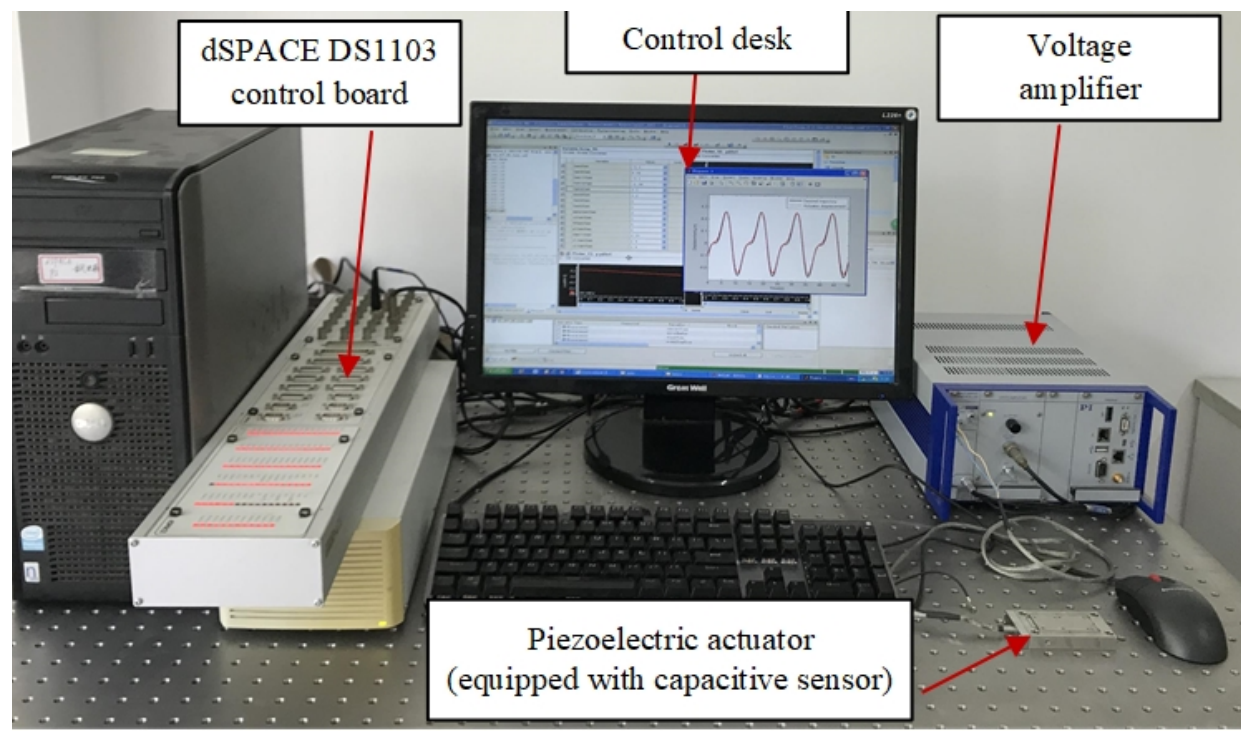

Figure 7. Experimental system.

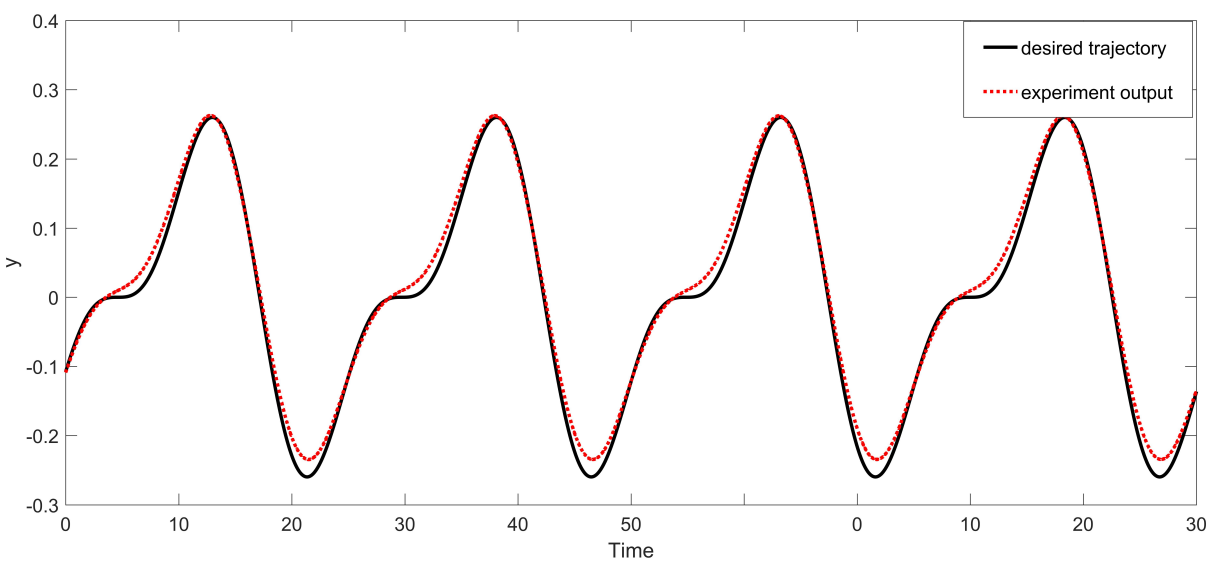

Figure 8. Experimental output-tracking performance.

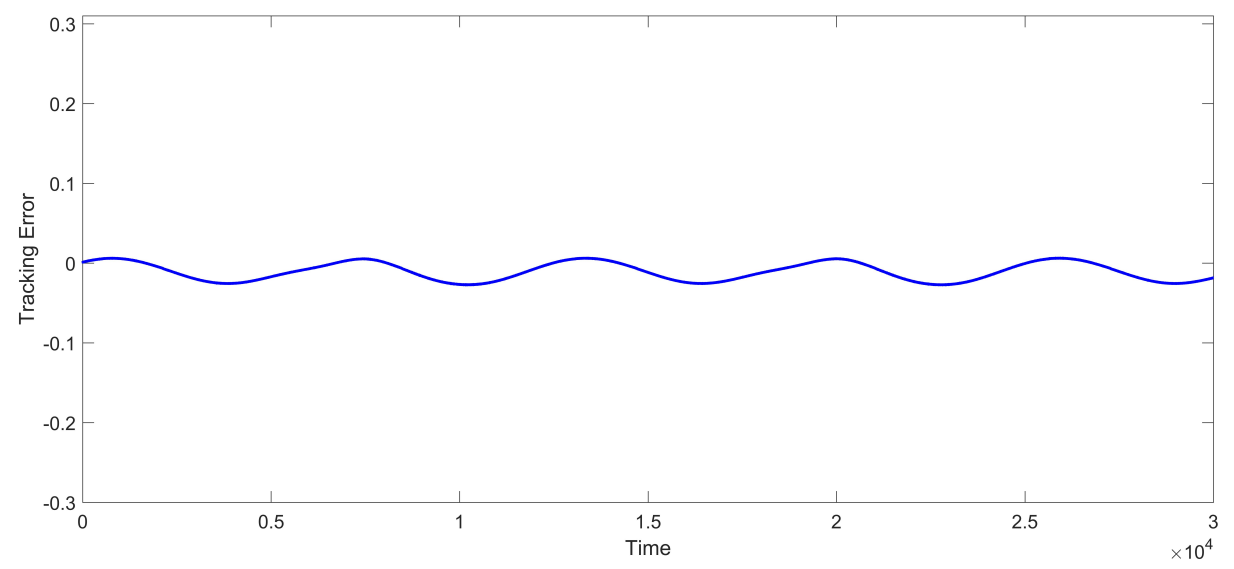

Figure 9. Error between the output of the experimental results and the desired trajectory.

\section{Conclusions}

Adaptive control is proposed in this work for an uncertain pure-feedback nonlinear system with asymmetric hysteresis. The asymmetric property of hysteresis nonlinearity is depicted through the introduction of an asymmetric item into the classical Bouc-Wen model. The novel control method 
is developed on the basis of nonaffine functions and backstepping technique to address nonaffine appearance of the system. Lyapunov analysis proves that all the signals in the closed loop ultimately guarantee global stability. The simulation comparison shows the effectiveness and superiority of the proposed control design, and the simulation results and dSPACE experimental work prove the feasibility and credibility of the proposed scheme.

Author Contributions: Conceptualization, X.L. and X.Z.; investigation, X.L.; project administration, X.L. and X.Z.; software, X.L.; supervision, H.P. and X.Z.; writing-original draft, X.L.; writing-review and editing, X.L. and X.Z.

Funding: This research was partially supported by the national natural science foundation of China (61273184).

Acknowledgments: The authors wish to express their gratitude to the support of the national natural science foundation of China (61273184).

Conflicts of Interest: The authors declare no conflict of interest.

\section{Appendix A}

Proof of the upper bound of $h$

Consider all the situations of the signs of $v, \dot{v}$ and $h$ as Table A1:

Table A1. Possibilities of the signs of $v, \dot{v}$, and $h$.

\begin{tabular}{cccc}
\hline Situation & $\boldsymbol{v}$ & $\dot{\boldsymbol{v}}$ & $\boldsymbol{h}$ \\
\hline 1 & + & + & + \\
2 & + & + & - \\
3 & + & - & + \\
4 & + & - & - \\
5 & - & + & + \\
6 & - & + & - \\
7 & - & - & + \\
8 & - & - & - \\
\hline
\end{tabular}

Define Lyapunov function $V=\frac{1}{2} h^{2}$. In situation $1,\left.\dot{V}\right|_{1}=h\left[\dot{v}\left(A_{b w}-h^{m}(\beta+\gamma)\right)+\delta \dot{v}\right] \leq 0$ for $h \geq \sqrt[m]{\frac{A_{b w}+\delta}{\beta+\gamma}}$. In situation $2, \dot{V} \mid s_{2}=h\left[\dot{v}\left(A_{b w}-|h|^{m}(-\beta+\gamma)\right)+\delta \dot{v}\right] \leq 0$ for all values of $h$. In situation $3, \dot{V} \mid s_{3}=h\left[\dot{v}\left(A_{b w}-h^{m}(-\beta+\gamma)\right)+\delta \dot{v}\right] \leq 0$ for all values of $h$. In situation $4, \dot{V} \mid s_{4}=h\left[\dot{v}\left(A_{b w}-\right.\right.$ $\left.\left.|h|^{m}(\beta+\gamma)\right)+\delta \dot{v}\right] \leq 0$ for $|h| \geq \sqrt[m]{\frac{A_{b w}+\delta}{\beta+\gamma}}$. In situation $5, \dot{V} \mid S_{5}=h\left[\dot{v}\left(A_{b w}-|h|^{m}(\beta+\gamma)\right)-\delta \dot{v}\right] \leq 0$ for $h \geq \sqrt[m]{\frac{A_{b w}-\delta}{\beta+\gamma}}$. In situation $6,\left.\dot{V}\right|_{s_{6}}=h\left[\dot{v}\left(A_{b w}-|h|^{m}(-\beta+\gamma)\right)-\delta \dot{v}\right] \leq 0$ for all values of $h$. In situation $7, \dot{V} \mid s_{7}=h\left[\dot{v}\left(A_{b w}-h^{m}(-\beta+\gamma)\right)-\delta \dot{v}\right] \leq 0$ for all values of $h$. In situation $8, \dot{V} \mid s_{8}=$ $h\left[\dot{v}\left(A_{b w}-|h|^{m}(\beta+\gamma)\right)-\delta \dot{v}\right] \leq 0$ for $|h| \geq \sqrt[m]{\frac{A_{b w}-\delta}{\beta+\gamma}}$.

Therefore, for all situations of the signs of $v, \dot{v}$ and $h, \dot{V} \leq 0$ for all $|h| \geq h_{0} \sqrt[m]{\frac{A_{b v v}-\delta}{\beta+\gamma}}$. Using [52], for every initial condition $h\left(t_{0}\right)$ and $h_{0} \sqrt[m]{\frac{A_{b w}-\delta}{\beta+\gamma}}$, it comes that $h \leq \max \left\{\left|h\left(t_{0}\right)\right|, h_{0}\right\}$. Therefore, the proof is completed.

\section{References}

1. Chouza, A.; Barambones, O.; Calvo, I. Sliding mode-based robust control for piezoelectric actuators with inverse dynamics estimation. Energies 2019, 12, 943. [CrossRef]

2. Tao, G.; Kokotovic, P.V. Adaptive control of plants with unknown hysteresis. IEEE Trans. Autom. Control 1995, 40, 200-212. [CrossRef]

3. Jang, M.J.; Chen, C.L.; Lee, J.R. Modeling and control of a piezoelectric actuator driven system with asymmetric hysteresis. J. Franklin Inst. 2009, 346, 17-32. [CrossRef]

4. Aguirre, G.; Janssens, T.; Brussel, H.V.; Al-Bender, F. Asymmetric-hysteresis compensation in piezoelectric actuators. Mech. Syst. Signal Process. 2012, 30, 218-231. [CrossRef] 
5. Gu, G.Y.; Zhu, L.M.; Su, C.Y.; Ding, H.; Fatikow, S. Modeling and control of piezo-actuated nanopositioning stages: A survey. IEEE Trans. Autom. Sci. Eng. 2016, 13, 313-332. [CrossRef]

6. Do, T.N.; Tjahjowidodo, T.; Lau, M.W.S. Hysteresis modeling and position control of tendon-sheath mechanism in flexible endoscopic systems. Mechatronics 2014, 24, 12-22. [CrossRef]

7. Kuhnen, K. Modeling, identification and compensation of complex hysteretic nonlinearities: A modified Prandtl-Ishlinskii approach. Eur. J. Control 2003, 9, 407-418. [CrossRef]

8. Jiang, H.; Ji, H.; Qiu, J.; Chen, Y. A modified Prandtl-Ishlinskii model for modeling asymmetric hysteresis of piezoelectric actuators. IEEE Trans. Ultrason. Ferroelectr. Freq. Control 2010 57, 1200-1210. [CrossRef]

9. Liu, S.; Su, C.Y. A note on the properties of a generalized PrandtlIshlinskii model. Smart Mater. Struct. 2011, 20, 0870031-0870037. [CrossRef]

10. Gu, G.Y.; Zhu, L.M.; Su, C.Y. Modeling and compensation of asymmetric hysteresis nonlinearity for piezoceramic actuators with a modified Prandtl-Ishlinskii model. IEEE Trans. Ind. Electron. 2014, 61, 1583-1595. [CrossRef]

11. Song, J.; Der Kiureghian, A. Generalized Bouc-Wen model for highly asymmetric hysteresis. J. Eng. Mech. 2006, 132, 610-618. [CrossRef]

12. Sireteanu, T; Giuclea, M ; Serban, V. On The fitting of experimental hysteretic loops by Bouc-Wen model. In Proceedings of the Annual Symposium of the Institute of Solid Mechanics SISOM, Bucharest, Romania, 29-30 May 2008; pp. 29-30.

13. Guo, Y.X.; Mao, J.Q. Modeling of hysteresis nonlinearity based on generalized Bouc-Wen model for GMA. In International Conference on Intelligent Robotics and Applications; Springer: Berlin/Heidelberg, Germany, 2010; pp. 147-158.

14. Su, C.Y.; Stepanenko, Y; Svoboda, J. Robust adaptive control of a class of nonlinear systems with unknown backlash-like hysteresis. IEEE Trans. Autom. Control 2000, 45, 2427-2432. [CrossRef]

15. Zhou, J.; Wen, C.; Zhang, Y. Adaptive backstepping control of a class of uncertain nonlinear systems with unknown backlash-like hysteresis. IEEE Trans. Autom. Control 2004, 49, 1751-1759. [CrossRef]

16. Su, C.Y.; Wang, Q.; Chen, X. Adaptive variable structure control of a class of nonlinear systems with unknown Prandtl-Ishlinskii hysteresis. IEEE Trans. Autom. Control 2005, 50, 2069-2074.

17. Wang, Q.; Su, C.Y. Robust adaptive control of a class of nonlinear systems including actuator hysteresis with Prandtl-Ishlinskii presentations. Automatica 2006, 42, 859-867. [CrossRef]

18. Ren, B.; Ge, S.S.; Lee, T.H.; Su, C.Y. Adaptive neural control for a class of nonlinear systems with uncertain hysteresis inputs and time-varying state delays. IEEE Trans. Neural Netw. 2009, 20, 1148-1164. [CrossRef]

19. Zhou, J.; Wen, C.; Li, T. Adaptive output feedback control of uncertain nonlinear systems with hysteresis nonlinearity. IEEE Trans. Autom. Control 2012, 57, 2627-2633. [CrossRef]

20. Liu, Y.H.; Feng, Y.; Chen, X. Robust adaptive dynamic surface control for a class of nonlinear dynamical systems with unknown hysteresis. Abstr. Appl. Anal. 2014, 2014, 640249. [CrossRef]

21. Chen, M.; Ge, S.S. Adaptive neural output feedback control of uncertain nonlinear systems with unknown hysteresis using disturbance observer. IEEE Trans. Ind. Electron. 2015, 62, 7706-7716. [CrossRef]

22. Lv, W.; Wang, F. Finite-time adaptive fuzzy tracking control for a class of nonlinear systems with unknown hysteresis. Int. J. Fuzzy Syst. 2018, 20, 782-790. [CrossRef]

23. Huo, X.; Ma, L.; Zhao, X.; Niu, B.; Zong, G. Observer-based adaptive fuzzy trackincontrol of MIMO switched nonlinear systems preceded by unknown backlash-like hysteresis. Inf. Sci. 2019, 490, 369-386. [CrossRef]

24. Chen, X.; Hisayama, T.; Su, C.Y. Pseudo-inverse-based adaptive control for uncertain discrete time systems preceded by hysteresis. Automatica 2009, 45, 469-476. [CrossRef]

25. Xu, Q.; Wong, P.K. Hysteresis modeling and compensation of a piezostage using least squares support vector machines. Mechatronics 2011, 21, 1239-1251. [CrossRef]

26. Feng, Y.; Rabbath, C.A.; Hong, H.; Su, C.Y.; Lin, W. Inverse hysteresis control for shape memory alloy micro-actuators based flap positioning system. In Proceedings of the IEEE Conference on Decision and Control, Atlanta, GA, USA, 15-17 December 2010; pp. 3662-3667.

27. Zhang, X.; Lin, Y.; Mao, J. A robust adaptive dynamic surface control for a class of nonlinear systems with unknown Prandtl-Ishilinskii hysteresis. Int. J. Robust Nonlinear Control 2011, 21, 1541-1561. [CrossRef]

28. Ren, B.; San, P.; Ge, S.S.; Lee, T.H. Adaptive dynamic surface control for a class of strict-feedback nonlinear systems with unknown backlash-like hysteresis. In Proceedings of the 2009 American Control Conference, St. Louis, MO, USA, 10-12 June 2009; pp. 4482-4487. 
29. Liu, Z.; Lai, G.; Zhang, Y.; Chen, X.; Chen, C.L. Adaptive neural control for a class of nonlinear time-varying delay systems with unknown hysteresis. IEEE Trans. Neural Netw. Learn. Syst. 2014, 25, 2129-2140.

30. Si, W.J.; Dong, X.D.; Yang, F.F. Adaptive neural prescribed performance control for a class of strict-feedback stochastic nonlinear systems with hysteresis input. Neurocomputing 2017, 251, 35-44. [CrossRef]

31. Huang, J.; Song, Y.D.; Wang, W.; Wen, C.; Li, G. Fully distributed adaptive consensus control of a class of high-order nonlinear systems with a directed topology and unknown control directions. IEEE Trans. Cybern. 2018, 48, 2349-2356. [CrossRef]

32. Huang, J.; Wang, W.; Wen, C.; Zhou, J. Adaptive control of a class of strict-feedback time-varying nonlinear systems with unknown control coefficient. Automatica 2018, 93, 98-105. [CrossRef]

33. Huang, J.; Wang, W.; Wen, C.; Li, G. Adaptive Event-Triggered Control of Nonlinear Systems with Controller and Parameter Estimator Triggering. IEEE Trans. Autom. Control 2019. [CrossRef]

34. Ramezan, Z.; Arefi, M.M.; Zargarzadeh, H.; Jahed-Motlagh, M.R. Neuro-adaptive backstepping control of SISO nonaffine systems with unknown gain sign. ISA Trans. 2016, 65, 199-209. [CrossRef]

35. Liu, Y.J.; Wang, Z.F. Adaptive fuzzy controller design of nonlinear systems with unknown gain sign. Nonlinear Dyn. 2009, 58, 687-695. [CrossRef]

36. Labiod, S.; Guerra, T.M. Indirect adaptive fuzzy control for a class of nonaffine nonlinear systems with unknown control directions. Int. J. Control Autom. Syst. 2010, 8, 903-907. [CrossRef]

37. Zhang, T.P.; Shi, X.C.; Zhu, Q.; Yang, Y.Q. Adaptive neural tracking control of pure-feedback nonlinear systems with unknown gain signs and unmodeled dynamics. Neurocomputing 2013, 121, 290-297. [CrossRef]

38. Boulkroune, A.; M'Saad, M.; Farza, M. Adaptive fuzzy tracking control for a class of MIMO nonaffine uncertain systems. Neurocomputing 2012, 93, 48-55. [CrossRef]

39. Park, J.H.; Park, G.T. Robust adaptive fuzzy controller for nonaffine nonlinear systems with dynamic rule activation. Int. J. Robust Nonlinear Control 2003, 13, 117-139. [CrossRef]

40. Ge, S.S.; Wang, C. Adaptive NN control of uncertain nonlinear pure-feedback systems. Automatica 2002, 38, 671-682. [CrossRef]

41. Na, J.; Ren, X.M.; Shang, C.; Gao, Y. Adaptive neural network predictive control for nonlinear pure feedback systems with input delay. J. Process Control 2012, 22, 194-206. [CrossRef]

42. Liu, Y.J.; Wang, W. Adaptive fuzzy control for a class of uncertain nonaffine nonlinear systems. Inf. Sci. 2007, 177, 3901-3917. [CrossRef]

43. Liu, Y.J.; Wang, W.; Tong, S.C. Adaptive fuzzy output tracking control for a class of uncertain nonlinear systems. Fuzzy Sets Syst. 2009, 160, 2727-2754. [CrossRef]

44. Zhang, Q.; Wang, C.; Xu, D. Finite-time stabilization for a class of nonaffine nonlinear systems with input saturation and time-varying output constraints. IEEE Access 2018, 6, 23529-23539. [CrossRef]

45. Fortuna, L.; Muscato, G. A roll stabilization system for a monohull ship: modeling, identification, and adaptive control. IEEE Trans. Control Syst. Technol. 1996, 4, 18-28. [CrossRef]

46. Wang, H.; Liu, P. X.; Niu, B. Robust fuzzy adaptive tracking control for nonaffine stochastic nonlinear switching systems. IEEE Trans. Cybern. 2017, 48, 2462-2471. [PubMed]

47. Charalampakis, A.E.; Dimou, C.K. Identification of Bouc-Wen hysteretic systems using particle swarm optimization. Comput. Struct. 2010, 88, 1197-1205. [CrossRef]

48. Charalampakis, A.E.; Koumousis, V.K. Identification of Bouc-Wen hysteretic systems by a hybrid evolutionary algorithm. J. Sound Vib. 2008, 314, 571-585. [CrossRef]

49. Polycarpou, M. M.; Ioannou, P. A. A robust adaptive nonlinear control design Automatica 1996, 32, $423-427$. [CrossRef]

50. Pettersen, K.Y. Lyapunov sufficient conditions for uniform semiglobal exponential stability. Automatica 2017, 78, 97-102. [CrossRef]

51. Liu, Y.H.; Huang, L.; Xiao, D.; Guo, Y. Global adaptive control for uncertain nonaffine nonlinear hysteretic systems. ISA Trans. 2015, 58, 255-261. [CrossRef]

52. Naser, M.; Fuad, M.; Ikhouane, F. Consistency of the Duhem model with hysteresis. Math. Prob. Eng. 2013. [CrossRef]

(C) 2019 by the authors. Licensee MDPI, Basel, Switzerland. This article is an open access article distributed under the terms and conditions of the Creative Commons Attribution (CC BY) license (http://creativecommons.org/licenses/by/4.0/). 